\author{
Original Research \\ Prosthetics and Orthotics International
}

Submitted 1.12.2017

Submitted revision 16.6.2018

Accepted 15.7.2018

\title{
Does an inverted pendulum model represent the gait of individuals with unilateral transfemoral amputation while walking over level ground?
}

Short title: Inverted pendulum gait model for individuals with UTF amputation Gerda $\underline{\text { Strutzenberger }}^{\mathrm{a}}$, Nathalie $\underline{\text { Alexander }}^{\mathrm{a}}$, Alan R. De Asha ${ }^{\mathrm{b}}$, Hermann $\underline{\text { Schwameder }}^{\mathrm{a}}$, Cleveland T. $\underline{\text { Barnett }}^{\mathrm{c}}$

${ }^{a}$ Department of Sport Science and Kinesiology, University of Salzburg, Schlossallee 49

5400 Hallein-Rif, Austria, gerda.strutzenberger@sbg.ac.at; nathalie.alexander@sbg.ac.at, hermann.schwameder@sbg.ac.at

${ }^{\mathrm{b} C-M o t i o n, ~ I n c .,} 20030$ Century Blvd. Suite 104A, Germantown, MD 20874, USA, alan.deasha@c-motion.com

${ }^{\mathrm{c}}$ School of Science and Technology, Nottingham Trent University, Nottingham, NG11 8NS, UK, cleveland.barnett@ntu.ac.uk

underscored: Last name of authors.

\section{Conflict of Interest Disclosure: None.}

\section{Corresponding Author:}

Gerda Strutzenberger, Department of Sport Science and Kinesiology, University of Salzburg, Schlossallee 49, 5400 Hallein, Austria. Tel.: 004366280444875

E-Mail address: gerda.strutzenberger@sbg.ac.at 


\section{Abstract}

\section{Study Design: Controlled Trial}

4 Background: An inverted pendulum model represents the mechanical function of able-bodied

5 walking accurately, with centre of mass height and forward velocity data plotting as sinusoidal

6 curves, 180 degrees out of phase.

7 Objectives: The current study investigated whether the inverted pendulum model represented

8 level gait in individuals with a unilateral transfemoral amputation.

9 Methods: Kinematic and kinetic data from ten individuals with unilateral transfemoral

10 amputation and fifteen able-bodied participants were recorded during level walking.

11 Results: During level walking, the inverted pendulum model described able-bodied gait well

12 throughout the gait cycle, with median relative time shifts between centre of mass height and

13 velocity maxima and minima of between $1.2 \%-1.8 \%$ of gait cycle. In the group with unilateral

14 transfemoral amputation, the relative time shift was significantly increased during the prosthetic-

15 limb initial double-limb support phase by $6.3 \%$.

16 Conclusion: The gait of individuals with unilateral transfemoral amputation shows deviation

17 from a synchronous inverted pendulum model during prosthetic limb stance. The reported

18 divergence may help explain such individuals' increased metabolic cost of gait. Temporal

19 divergence of inverted pendulum behaviour could potentially be utilised as a tool to assess the

20 efficacy of prosthetic device prescription.

21 Word count: 191 
1 Clinical Relevance: The size of the relative time shifts between CoM height and velocity

2 maxima and minima could potentially be used as a tool to quantify the efficacy of innovative

3 prosthetic device design features aimed at reducing the metabolic cost of walking and improving

4 gait efficiency in individuals with amputation.

\section{Keywords:}

6 Centre of mass velocity, centre of mass height, mechanistic gait model 


\section{Background}

2 Researchers have previously used an inverted pendulum model to represent the mechanical

3 functioning of normal, bipedal overground walking (e.g. 1, 2-5). The inverted pendulum model

4 is, by intention, simplistic. It represents each of the lower limbs as a single rigid segment, capable

5 of rotation about the ankle during stance, with the whole-body centre of mass (CoM) located at

6 its superior end. This model sits comfortably with the mechanical reality of walking, as the

7 vertical trajectory of the $\mathrm{CoM}$ and its forward velocity vary during the gait cycle in a similar

8 manner to that of the bob of an oscillating pendulum. The CoM is higher and slower during

9 single-limb support and lower and faster during double-limb support. When plotted graphically,

10 CoM height and CoM forward velocity appear as sinusoidal curves, $180^{\circ}$ out of phase, with CoM

11 peak forward velocity coinciding with the lowest CoM height and CoM minimum forward

12 velocity coinciding with maximum CoM height.

13 Individuals with a unilateral lower-limb amputation, are mechanically constrained during walking

14 by the prosthetic device used (6). As a result, they e.g. walk more slowly (7), fall more often (8)

15 and have a higher metabolic cost of walking (9-11) when compared to able-bodied individuals.

16 Multiple issues may affect the gait pattern of any given population of individuals with lower-limb

17 amputation, such as e.g. the ability of the prosthetic ankle and/or knee joint to allow flexion

18 during stance. It is not well understood if the mechanical constraints imposed by prosthetic

19 devices mean a mechanical model of walking could be applicable to individuals with a lower-

20 limb amputation. It has been demonstrated not to be the case in individuals with a unilateral

21 transtibial amputation (UTT), where peak CoM forward velocity and minimum CoM height did

22 not coincide during prosthetic-limb initial double-limb support (12). In such individuals, the CoM 
1 accelerates forward to peak velocity after the minimum CoM height occurs, therefore whilst the

2 CoM is being elevated.

4 Specific gait characteristics have been reported for individuals with unilateral transfemoral 5 amputation (UTF). For example, those with UTF are likely to retain a more vertical projection of

6 the CoM at prosthetic-limb toe-off (13) and commonly use a vaulting mechanism, e.g. via

7 increased plantarflexion, during the intact-limb stance phase. This vaulting strategy supports the

8 elevation of the CoM and hence allows for sufficient prosthetic-limb toe clearance during swing

9 (14). Also, during the prosthetic-limb stance phase, prosthetic knee flexion is restricted in order

10 to prevent it unlocking and collapsing during weight bearing (15). Both aspects could affect the

11 efficacy of applying the inverted pendulum model to UTF gait, as the model does not include

12 varying pendulum lengths (as induced by the vaulting mechanism) and restrained knee flexion.

13 Additional the mechanical constraint to rotate around the flexion/extension axis of the ankle

14 might affect the synchrony of the inverted pendulum model . To date, the possibilities of applying

15 the inverted pendulum model to the gait of individuals with a UTF has not been established and it

16 remains unclear to what extent their specific gait characteristics influence the efficacy of applying

17 this model. Given the importance of step-to-step transitions in determining the metabolic cost of

18 walking $(5,11)$, and that disruptions of energy transformations associated with the inverted

19 pendulum mechanism can lead to increased energy expenditure (16), any breakdown of

20 compliance with the inverted pendulum model may partially explain the higher metabolic cost of

21 walking for those with a lower-limb amputation as demonstrated by Houdijk, Pollmann (11) for 22 individuals with UTT. 
1 Therefore, the objective of this study was to investigate whether the level ground gait of

2 individuals with UTF is represented by an inverted pendulum model . It was hypothesized that

3 the model would during intact-limb stance perform similar for individuals with UTF compared to

4 able-bodied individuals, but different during prosthetic-limb stance.

6 Methods

7 Study Design: As study design, a controlled trial was chosen, comparing able-bodied individuals

8 with individuals with a unilateral transfemoral amputation.

9 Participants: The UTF group consisted of ten participants, all using the same prosthetic foot

10 (Esprit; Chas. A. Blatchford \& Sons, Basingstoke, UK). Data from eight (Mean (SD) age: 42

11 (14.8) years, mass: $86.3(15.3) \mathrm{kg}$, height: $1.74(0.06) \mathrm{m}$.) walking over level ground, previously

12 published in De Asha, Munjal (12), were re-analyzed. Data from two further individuals with

13 UTF (Mean (SD) age: 53 (2.8) years, mass: $74.1(14.9) \mathrm{kg}$, height: $1.81(0.11) \mathrm{m}$ ) and 15 able-

14 bodied (AB) male individuals (Mean (SD) age: 27 (5) years, mass: $75.1(9.1) \mathrm{kg}$, height: 1.81

$15(0.05) \mathrm{m})$ were collected at the University of Salzburg. All UTF participants had undergone

16 amputation at least two years prior to participation, were free from neurological, musculoskeletal

17 (other than limb amputation) or cardiovascular disorders, and had used their current prostheses

18 for at least six months. All were classed as being at least K3 on the Medicare scale by their

19 prescribing clinician and all used their habitual knee component (1x Orion, 1x EUK SAKL, 2x

20 KX06: all Chas A Blatchford \& Sons, Basingstoke, UK; 1x C-leg, 1x 3R45: both Otto Bock,

21 Duderstadt, Germany; and 3x Total Knee: Ossur, Reykjavik, Iceland). Institutional bioethics 
1 committee approval was obtained at both locations and written informed consent was provided by

2 all participants prior to data collection.

3 Data acquisition: In addition to the data from De Asha, Munjal (12), kinematic and kinetic data

4 were collected at $250 \mathrm{~Hz}$ and $1000 \mathrm{~Hz}$ respectively using an eight camera motion capture system

5 (Vicon, Oxford, UK) and two force plates (AMTI, Watertown, MA, USA). Participants walked at

6 their self-selected walking speed on an $8 \mathrm{~m}(8 \mathrm{TF}, 12)$ and $6 \mathrm{~m}(2 \mathrm{TF}$ all AB, Salzburg) walkway

7 with the two force platforms embedded in the middle of the walkways.

8 Reflective markers were attached to participants according to the Cleveland Clinic Marker Set

9 (Motion Analysis Corp, Santa Rosa, USA), which uses an anatomical landmark calibration

10 technique (17). Markers on the prosthetic knee were placed medially and laterally of the

11 rotational or approximated (for polycentric knee componentes) joint centre. At the prosthetic

12 ankle and foot, the markers were placed to correspond with those on the intact side. For all UTF

13 participants the mean of six trials at self-selected walking speed were analysed, while for the $\mathrm{AB}$

14 group one representative trial was analysed for each participant.

15 Data processing: Labeling of marker trajectories was undertaken in Workstation and Nexus

16 software (Vicon, Oxford, UK) before all data were exported to Visual3D (C-Motion,

17 Germantown, USA) from which point on the processing was identical for all participants.

18 Kinematic and kinetic data were filtered using a fourth order, zero-lag Butterworth filter with a 6

$19 \mathrm{~Hz}$ cut-off. A nine segment model (head, trunk, pelvis, thighs, shanks and feet) of each

20 participant was constructed. Initial contact (IC) and toe off (TO) were defined as the instants the

21 vertical component of the ground reaction force first went above or below $20 \mathrm{~N}$, respectively. The

22 gait cycle was defined from IC of the prosthetic (UTF) or right (AB) limb to the consecutive IC 
1 of the ipsilateral limb and normalized to $100 \%$. In instances where no kinetic data were available

2 (i.e. a missed or incomplete force platform contact), IC and TO were defined using kinematic

3 data $(18,19)$. From these events phases of single-limb support(SS) and double-limb support

4 (DS), were defined. The position of the whole-model CoM was calculated as the weighted 5 average of the model's segmental CoMs (20-22). Forward velocity of the CoM was defined as

6 the first derivation of the CoM position in the walking direction, which was in line with the

7 global coordinate system of the respective laboratory. The height of the CoM was defined as the

8 instantaneous vertical distance between the CoM and the walking surface. For the UTF group

9 timings, normalised to the gait cycle, of the minimum and maximum CoM height and forward

10 velocity were identified in the following four phases: (i) Prosthetic initial double-support

11 (IDS_P); (ii) Prosthetic single-support (SS_P); (iii) Prosthetic terminal double-support (TDS_P);

12 (iv) Intact single-support (SS_I) (Figure 1). For the AB group these timings were identical, only

13 the analyzed gait cycle started with the right foot always, resulting in prosthetic phases

14 corresponding to the right side and SS_I corresponding to the left side (Figure 1).

16 For each phase, the relative time shift was calculated as the time difference between the maxima

17 and minima of CoM vertical position and forward velocity normalised to gait cycle duration.

18 Assuming inter-limb symmetry in the $\mathrm{AB}$ group, the mean of both, left and right, limbs were

19 calculated for the metric values. In case of perfect compliance with the inverted pendulum, a time

20 shift of zero would be observed with increasing time shift magnitude indicating reduced

21 adherence to the inverted pendulum model. 
1 Statistical analysis: Statistical testing was performed_using SPSS software (v.23, IBM, Armonk,

2 NY, USA). UTF data were not normally distributed, thus a non-parametric statistical approach

3 was adopted for all participants.

4 The data first was analyzed to detect main effect of groups (UTF and AB) using a Friedman

5 Analysis of Variances by ranks . A second Friedman Analysis of Variances by ranks was used to

6 detect the main effect of phases (IDS_P, SS_P, TDS_P, SS_I). Post-hoc tests were conducted

7 where significant main effects were identified using a) for each phase the Mann-Whitney U-test

8 to detect differences between groups (AB vs. UTF) and b) for each group the Wilcoxon signed

9 rank test to detect differences between the phases ). For all statistical tests an alpha level of 5\%

$10(p<0.05)$ was set. Post-hoc test were conducted using a Bonferroni-corrected alpha of $\mathrm{p}=0.008$

11 for phase comparisons, and $\mathrm{p}=0.013$ for group comparisons. Effect sizes were calculated as $\mathrm{r}$

12 (23) with the boundaries of $0.1,0.3$ and 0.5 for small, medium and large.

\section{Results}

15 During level walking there were significant effects of group $(\mathrm{p}=0.004)$, gait cycle phase $(\mathrm{p}<$

16 0.001) and a group by phase interaction $(\mathrm{p}<0.001)$ on the relative timings of CoM height and

17 velocity maxima and minima. Between gait phases the $\mathrm{AB}$ group did not show significant

18 differences in time shifts between CoM maxima and minima of height and forwards velocity,

19 with median relative time shifts ranging between $1.2 \%$ and $1.8 \%$ of gait cycle duration $(p>$

20 0.087, Figure $1 \& 2$ ). For the UTF group the median relative time shift was significantly

21 increased during IDS_P phases compared to the TDS_P $(p=0.005, \mathrm{r}=0.89)$ and SS_I phase $(p=$

$220.005, \mathrm{r}=0.89$ ) (Figure 1). Post hoc analysis of differences between the UTF and the AB group 
1 indicated the difference was only significant during the IDS_P phase, when the UTF group

2 displayed increased relative time shifts compared to the AB group (IDS_P, $p<0.001, \mathrm{r}=0.79$ ),

3 indicating that maximum CoM velocity occurred after minimum CoM height. In the TDS_P

4 double-limb support phase the maximum CoM velocity was almost synchronous with minimum

5 CoM height (Table 1, Figure 2).

6

7

8 Table 1: Median (IQR) of relative time shift of unilateral transfemoral (UTF) and able-bodied

9 (AB) participants during initial and terminal double and single-limb support phases in level gait

\begin{tabular}{cccccc} 
& Phase & AB & UTF & $\begin{array}{c}\text { p-value } \\
\text { AB-UTF }\end{array}$ & $\begin{array}{c}\text { ES r } \\
\text { AB-UTF }\end{array}$ \\
\hline & IDS_P & $1.2(1.5)$ & $6.3(4.8)$ & $0.000^{*}$ & 0.79 \\
relative time shift [\%] & SS_P & $1.8(2.6)$ & $5(5.8)$ & 0.026 & 0.44 \\
& TDS_P & $1.2(1.5)$ & $-0.5(4.1)$ & 0.318 & 0.2 \\
& SS_I & $1.8(2.6)$ & $3.7(2)$ & 0.028 & 0.44 \\
\hline
\end{tabular}

IDS_P: Initial double-limb support, prosthetic-limb leading limb, SS_P: Single-limb support with prosthetic-limb, TDS_P: Terminal double-limb support, prosthetic-limb tailing limb, SS_I: Single-limb support with intact-limb AB: able-bodied group, UTF: group with unilateral transfemoral amputation, ES r: $\quad$ Effect size

* indicates significant difference between AB and UTF group, Bonferroni-corrected $\mathrm{p}=0.013$

12

\section{Discussion}

14 The aim of the current study was to assess the efficacy of the application of an inverted pendulum

15 model to the overground gait of individuals with UTF. The prosthetic limb of individuals with

16 UTF more closely replicates the characteristics of a rigid segment than does an intact leg, due

17 primarily to the non-articulation or reduced articulation of the prosthetic knee during stance and

18 also the mechanical nature of prosthetic componentry making up a large proportion of the 
1 affected limb. However constrained movement in the prosthetic ankle joint may act against the

2 inverted pendulum model. Therefore, it is not intuitively clear, whether the inverted pendulum

3 model would represent UTF gait well. The results of the current study partially support the

4 hypothesis that the inverted pendulum model would be applied similarly to both $\mathrm{AB}$ gait and

5 UTF gait during intact-limb stance, but less so during prosthetic-limb stance. As hypothesised,

6 the magnitude of relative time shifts between CoM height and velocity maxima and minima in

7 the $\mathrm{AB}$ group were consistently close to zero across all phases of the gait cycle, indicating that an

8 inverted pendulum model is a reasonable estimation of AB gait. In the UTF group, the relative

9 time shifts were not consistent across all phases of the gait cycle and showed an increased

10 relative time shift for IDS_P, during which the prosthetic-limb was being loaded, compared to the

11 gait phases in which the prosthetic-limb was in single-limb support, terminal double-limb

12 support, or in swing. During IDS_P, the maximum CoM forwards velocity occurred after the

13 minimum CoM height, as has been reported previously in individuals with UTT (12). The IDS_P

14 was the only phase showing a statistically significant increase in relative time shift between the

15 CoM height and forward velocity maxima and minima for the UTF group, compared to the AB

16 group. This suggested that whilst an inverted pendulum model of gait could be applied

17 appropriately to AB gait, this was not generally the case in the UTF group, especially during the

18 IDS_P phase. However, the lowest observed relative time shift in the UTF group also occurred

19 during prosthetic-limb stance, during TDS_P. During TDS_P, UTF gait appeared to function in

20 accordance with the inverted pendulum model in a way most like that of the AB group, with

21 maximum CoM velocity occurring almost synchronously with minimum CoM height (median 
1 difference $-0.5 \%$ gait cycle), therefore the hypothesis that the model would perform less well

2 during prosthetic stance was only partially supported.

3 The results from the current study are similar to that of previous research, where the application

4 of an inverted pendulum model to the gait of individuals with UTT was found to be inappropriate

5 (12). The current study demonstrates that for individuals with UTF, during the IDS_P phase

6 when the prosthetic-limb is being loaded, significant temporal separations between CoM

7 maximum velocity and minimum height occur. This can possibly be explained by an altered

8 loading response knee flexion pattern induced by the prosthetic knee type resulting in minimum

9 CoM height occurring earlier than in the $\mathrm{AB}$ group. If this was due to the possible use of the

10 controlled stance phase flexion provided by 7 out of the 10 knee types or due to a locked knee

11 during stance phase cannot be answered by this study. Thus peak CoM velocity is observed

12 whilst the CoM is being elevated in individuals with UTF. Conversely, the two events are

13 coincident in $\mathrm{AB}$ individuals, as loading response knee flexion appears to extend the $\mathrm{CoM}$

14 lowering phase in order to allow minimum CoM height to coincide with CoM peak forwards

15 velocity (Figure 1). Similarly, the same direction of temporal offset between minimum CoM

16 height and peak CoM velocity observed in UTT participants, where the CoM is accelerating

17 forwards while it is being elevated (12), may be due to reduced loading response flexion, which

18 typically occurs in such individuals because of impingement of the knee by the socket and the

19 desire to reduce in-socket/residuum torques. Given that both UTF and UTT participants have

20 similar relative time shifts between maximal and minimal CoM displacements and velocities

21 during IDS_P, it would appear reasonable that restricted loading response knee flexion could, at

22 least partially, be an explanation for this temporal offset. 
1 During the transition from one leg to the other in $\mathrm{AB}$ gait, progression is typically accomplished

2 via positive work through ankle plantarflexion of the trailing leg. Individuals with lower-limb

3 amputation cannot actively generate power at the prosthetic ankle, and hence use a more

4 energetically costly strategy involving positive work at the residual hip during early intact-limb

5 stance $(2,24)$. All participants in the current study used the same, non-articulating energy storing

6 and returning prosthetic foot device, so (lack of) power generation at the prosthetic ankle was

7 likely similar for all. However, the UTF participants were most divergent from the inverted

8 pendulum model during IDS_P, when the intact-limb was pushing off. This suggests that it is the

9 leading, weight accepting limb that modulates the timing of CoM maximum velocity and

10 minimum height during double-limb support and ultimately, adherence to the inverted pendulum

11 model of gait. While a large body of research focuses on the (lack of) propulsion provided during

12 TDS_P, the results of the current study point at the reduction in passive and eccentric work

13 during IDS_P also being an important factor in the higher metabolic cost of gait experienced by

14 individuals with lower-limb amputation. Increased energy expenditure has been proposed to be

15 associated with disruptions in energy transformations within the inverted pendulum model (16),

16 which would support this supposition. There are no supporting data, but our suggestion would be

17 that while a lack of active work at the prosthetic 'ankle' during late stance undoubtedly

18 contributes to the previously reported increased metabolic cost of walking in individuals with

19 lower limb amputation $(9,10)$, the lack of passive, or eccentric, energy absorption during early

20 and mid-stance does so too. This suggestion is supported by a previous finding in individuals

21 with UTT, who experienced a significant reduction in mechanical work, per meter travelled, done

22 at the intact leg when they used a hydraulically articulating ankle foot device that absorbed more 
1 energy during early stance, and returned less energy during late stance, than their customary, non-

2 articulating prosthetic feet (25). This should certainly be the subject of future study.

3 Finally, as impairments in gait function seem to be reflected by compliance, or lack of, to the

4 inverted pendulum model, the resulting relative time shift might be of clinical relevance. For

5 example, the size of the relative time shifts between CoM height and velocity maxima and

6 minima could potentially be used as a tool to quantify the efficacy of innovative prosthetic device

7 design features aimed at reducing the metabolic cost of walking and improving gait efficiency in

8 individuals with amputation.

9 It has to be kept in mind, that while a high standardization of used components is wished for, it 10 often does not reflect reality. While all participants used the same type of ankle device, a

11 variation of knee components were used and possible individual specific gait patterns induced by

12 specific knee components were not considered. Additionally it has to be kept in mind, that the

13 results reflect the gait using a rigid ankle device which does not reflect the nature of the inverted

14 pendulum as such that the pendulum pivots over a rotation center, which could also explain the

15 increased time shift in the initial prosthetic double support phase.

16 In conclusion results from the current study demonstrate that during level walking, an inverted

17 pendulum model represents $\mathrm{AB}$ gait well, but deviation to the model were seen in parts of the

18 UTF gait. In addition, UTF gait functioned mechanically most like AB gait during prosthetic-

19 limb single and terminal double-limb support and intact-limb single-limb support. Divergence

20 from inverted pendulum behaviour during the initial prosthetic double-limb support phase

21 following prosthetic-limb IC indicates that it is the loading limb that primarily modulates timing

22 of CoM maximum velocity and minimum height, rather than the unloading limb. These findings 
1 may also help explain the increased metabolic cost of walking, compared to the able-bodied, in

2 individuals with UTF. This relationship should be further investigated.

3 Word count: 2835

5 Acknowledgements

6 The authors would like to thank Endolite Deutschland GmbH (Kulmbach, Germany) for

7 providing the foot-component for the two UTF participants measured in Salzburg. Endolite

8 Deutschland $\mathrm{GmbH}$ had no role in the study conception and design; in the collection, analysis

9 and interpretation of data; in the writing of the manuscript; and in the decision to submit the

10 manuscript for publication.

\section{References}

13 1. Alexander RM. Simple models of human motion. Applied Mechanics Review. 14 1995;48:461-9.

15 2. Donelan JM, Kram R, Kuo AD. Simultaneous positive and negative external mechanical 16 work in human walking. J Biomech. 2002;35(1):117-24.

17 3. Donelan JM, Kram R, Kuo AD. Mechanical work for step-to-step transitions is a major 18 determinant of the metabolic cost of human walking. J Exp Biol. 2002;205(Pt 23):3717-27.

19 4. Kajita S, Kanehiro F, Fujiwara K, Yokoi K, Hirukawa H. Biped walking pattern 20 gerneation by a simple three-dimensional inverted pendulum model. Advanced Robotics. $21 \quad 2003 ; 17: 131-47$.

22 5. Kuo AD, Donelan JM, Ruina A. Energetic consequences of walking like an inverted 23 pendulum: step-to-step transitions. Exerc Sport Sci Rev. 2005;33(2):88-97.

24 6. Buckley JG, De Asha AR, Johnson L, Beggs CB. Understanding adaptive gait in lower25 limb amputees: insights from multivariate analyses. J Neuroeng Rehabil. 2013;10:98.

267 Waters RL, Perry J, Antonelli D, Hislop H. Energy cost of walking of amputees: the 27 influence of level of amputation. J Bone Joint Surg Am. 1976;58(1):42-6.

28 8. Kulkarni J, Wright S, Toole C, Morris J, Hirons R. Falls in patients with lower limb 29 amputations: prevalence and contributing factors. Physiotherapy. 1996;82:130-6. 
19 . Colborne GR, Naumann S, Longmuir PE, Berbrayer D. Analysis of mechanical and 2 metabolic factors in the gait of congenital below knee amputees. A comparison of the SACH and 3 Seattle feet. Am J Phys Med Rehabil. 1992;71(5):272-8.

4 10. Gailey RS, Wenger MA, Raya M, Kirk N, Erbs K, Spyropoulos P, et al. Energy 5 expenditure of trans-tibial amputees during ambulation at self-selected pace. Prosthet Orthot Int. 6 1994;18(2):84-91.

7 11. Houdijk H, Pollmann E, Groenewold M, Wiggerts H, Polomski W. The energy cost for 8 the step-to-step transition in amputee walking. Gait Posture. 2009;30(1):35-40.

9 12. De Asha AR, Munjal R, Kulkarni J, Buckley JG. Impact on the biomechanics of 10 overground gait of using an 'Echelon' hydraulic ankle-foot device in unilateral trans-tibial and trans-femoral amputees. Clin Biomech (Bristol, Avon). 2014;29(7):728-34.

13. Nolan L, Wit A, Dudzinski K, Lees A, Lake M, Wychowanski M. Adjustments in gait symmetry with walking speed in trans-femoral and trans-tibial amputees. Gait Posture. 2003;17(2):142-51.

14. Drevelle X, Villa C, Bonnet X, Loiret I, Fode P, Pillet H. Vaulting quantification during level walking of transfemoral amputees. Clin Biomech (Bristol, Avon). 2014;29(6):679-83.

15. Vrieling AH, van Keeken HG, Schoppen T, Otten E, Halbertsma JP, Hof AL, et al. Uphill and downhill walking in unilateral lower limb amputees. Gait Posture. 2008;28(2):235-42.

16. Ortega JD, Farley CT. Minimizing center of mass vertical movement increases metabolic cost in walking. J Appl Physiol (1985). 2005;99(6):2099-107.

17. Selbie WS, Hamill J, Kepple T. Chapter 7: Three Dimensional Kinetics. In: Robertson G, Caldwell G, Hamill J, Whittlesey S, editors. Research Methods in Biomechanics. 2nd edition ed. Champaign, IL, USA: Human Kinetics; 2013. p. 151-76.

18. De Asha AR, Robinson MA, Barton GJ. A marker based kinematic method of identifying initial contact during gait suitable for use in real-time visual feedback applications. Gait Posture. 2012;36(3):650-2.

19. Zeni JA, Jr., Richards JG, Higginson JS. Two simple methods for determining gait events during treadmill and overground walking using kinematic data. Gait Posture. 2008;27(4):710-4.

20. Hanavan E. A Mathematical Model for the Human Body. Wright-Patterson Air Force Base; 1964.

21. Vanrenterghem J, Gormley D, Robinson M, Lees A. Solutions for representing the wholebody centre of mass in side cutting manoeuvres based on data that is typically available for lower limb kinematics. Gait Posture. 2010;31(4):517-21.

22. Dempster WT. Space reqiuriements of the seated operator. Wright-Patterson Air Force Base, Ohio; 1995.

23. Cohen J. A power primer. Psychol Bull. 1992;112(1):155-9.

24. Kuo AD. Energetics of actively powered locomotion using the simplest walking model. J Biomech Eng. 2002;124(1):113-20.

25. De Asha AR, Munjal R, Kulkarni J, Buckley JG. Walking speed related joint kinetic alterations in trans-tibial amputees: impact of hydraulic 'ankle' damping. J Neuroeng Rehabil. 2013;10:107. 
1 Figure Headings

2 Figure 1: Mean forward CoM velocity (dotted lines) and CoM height relative to the CoM position

3 at initial contact (solid lines) for all AB (black lines) and UTF (grey lines) participants throughout

4 a normalised gait cycle during level walking. Initial and terminal Double-limb support (IDS,

5 TDS) and single-limb support (SS) phases are indicated by solid vertical lines, the relative time

6 shift between maximum and minimum positions is shown as shaded areas.

8 Figure 2: Boxplots of relative time shift between extreme values of CoM height and CoM 9 forward velocity in the initial and terminal double-limb support (IDS_P and TDS_P) and single10 limb support (SS_P and SS_I) phase of level gait.

11 Note: $\mathrm{UTF}=$ participant with unilateral transfemoral amputation, $\mathrm{AB}=$ able bodied participant,

12 IC = initial contact, TDS = terminal double-limb support, $\mathrm{P}=$ prosthetic-limb, $\mathrm{I}=$ intact-limb, $\mathrm{R}$

$13=$ right limb, $\mathrm{L}=$ left limb, * indicates significant difference $(p<0.050), \mathrm{r}=$ effect size, $\mathrm{s}=$ 14 small, $\mathrm{m}=$ medium, $1=$ large 